\title{
A SIMPLE CONSTRUCTION OF ROBINSON EQUIVALENCE RELATIONS
}

\author{
DANIELE MUNDICI
}

\begin{abstract}
We present the simplest constructions of nontrivial Robinson equivalence relations.
\end{abstract}

Several chapters of the book [1] deal with equivalence relations having the Robinson property (see Chapter XIX for definitions). For instance this property is naturally found in Keisler's probability logic (Chapter XIV), in topological and monotone logic (Chapters XV and III, via Chapter II, 7.1.5), and more generally, in nets of noncommutative Stone spaces $[\mathbf{7}, \mathbf{8}]$.

Since $\equiv_{\mathcal{L}_{\kappa \kappa}^{2}}$ for $\kappa$ extendible (Chapter XVIII, 3.3.7) had long been the only nontrivial example known to logicians, Lipparini's limit ultrapower characterization [3] yields the most general possible Robinson equivalence relation on the class of all (first-order) structures. This contrasts with the case of countable structures, where one only has the two trivial possibilities: elementary equivalence $\equiv$, and isomorphism $\cong[\mathbf{4}]$.

We aim at constructing large classes of examples, using only a minimum of the limit ultrapower machinery. We thus intend to provide raw material for the abstract theory developed in [5, 6]. As proved by Nadel [9], every equivalence relation $\sim$ (with the Robinson property) determines a smallest logic $L$ with $\equiv_{L}=\sim$, closed under atomic and Scott sentences, boolean operations (and obeying the Robinson consistency theorem). Hence our self-contained paper also contributes to the program of [9] of constructing logics with prescribed properties, starting from equivalence relations.

The author wishes to thank the referee for his suggestions and remarks.

THEOREM. There is a sequence $\left\{\sim_{\alpha} \mid \alpha \in O n\right\}$ of distinct Robinson equivalence relations with the additional property that for each ordinal $\alpha$ and each denumerable structure $\mathfrak{M}$ there is an uncountable structure $\mathfrak{N}$ such that $\mathfrak{M} \sim_{\alpha} \mathfrak{N}$.

ProOF. Referring throughout to [2], let $\pi=\pi(D, V)=\Pi_{D \mid V}$ be the limit ultrapower determined by the ultrafilter $D$ on the index set $I=\bigcup D$, and the filter $V$ on $I \times I$. Then $\pi$ is a unary operation on the class of all structures, and we let $\pi^{n}$ denote the $n$-fold composition of $\pi$, where $\pi^{0}=$ identity. Each $\pi^{n}$ is still a limit ultrapower. The class of all constructible limit ultrapowers can be naturally listed in a sequence indexed by the ordinals. This allows us to unambiguously speak of the first constructible limit ultrapower having a certain property. We define a sequence of triplets $\left\langle\lambda_{\alpha}, \pi_{\alpha}, \mu_{\alpha}\right\rangle_{\alpha \in O n}$ by the following stipulations, where $\mathbf{Z}$ is the set of integers (actually, any other denumerable set would do as well):

Received by the editors June 20, 1986.

1980 Mathematics Subject Classification (1985 Revision). Primary 03C95, 03C20, 03C40; Secondary $03 \mathrm{C} 07,03 \mathrm{C} 30,03 \mathrm{C} 52$. 
$\lambda_{0}=$ least cardinal $\lambda>\omega$ such that there is a constructible limit ultrapower $\pi$ satisfying $|\pi \mathbf{Z}|=\lambda$;

$\pi_{0}=$ first constructible limit ultrapower $\pi$ with $|\pi \mathbf{Z}|=\lambda_{0}$;

$\mu_{0}=\sup _{n \in \omega}\left|\pi_{0}^{n} \mathbf{Z}\right|$,

and for all ordinals $\alpha>0$,

$\lambda_{\alpha}=$ least cardinal $\lambda>\sup _{\rho<\alpha} \mu_{\gamma}$ such that there is a constructible limit ultrapower $\pi$ satisfying $|\pi \mathbf{Z}|=\lambda$;

$\pi_{\alpha}=$ first constructible limit ultrapower $\pi$ with $|\pi \mathbf{Z}|=\lambda_{\alpha} ;$

$\mu_{\alpha}=\sup _{n \in \omega}\left|\pi_{\alpha}^{n} \mathbf{Z}\right|$.

For each ordinal $\alpha$ we now define $\sim_{\alpha}$ by $\mathfrak{M} \sim_{\alpha} \mathfrak{N}$ iff for some $m, n \in \omega, \pi_{\alpha}^{m} \mathfrak{M} \cong$ $\pi_{\alpha}^{n} \mathfrak{N}$. It follows that $\sim_{\alpha}$ is an equivalence relation on the class of all structures, and whenever $\mathfrak{M} \sim_{\alpha} \mathfrak{N}$, then $\mathfrak{M}$ and $\mathfrak{N}$ necessarily have the same type. Clearly, $\sim_{\alpha}$ is preserved under the reduct and the renaming operations on structures. Further, $\cong$ is a refinement of $\sim_{\alpha}$, and $\sim_{\alpha}$ is a refinement of $\equiv$. We show that $\sim_{\alpha}$ has the Robinson property. Let the structures $\mathfrak{M}$ and $\mathfrak{N}$ have type $\tau_{\mathfrak{M}}$ and $\tau_{\mathfrak{N}}$ respectively; let $\tau=\tau_{\mathfrak{M}} \cap \tau_{\mathfrak{N}}$. Assume $\mathfrak{M} \backslash \tau \sim_{\alpha} \mathfrak{N} \uparrow \tau$, i.e. $\pi_{\alpha}^{m}(\mathfrak{M} \backslash \tau) \cong \pi_{\alpha}^{n}(\mathfrak{N} \uparrow \tau)$ for some $m, n \in \omega$. Then $\left(\pi_{\alpha}^{m} \mathfrak{M}\right) \backslash \tau \cong\left(\pi_{\alpha}^{n} \mathfrak{N}\right) \uparrow \tau$, and hence there exists a (unique, up to isomorphism) structure $\mathfrak{B}$ of type $\tau_{\mathfrak{M}} \cup \tau_{\mathfrak{N}}$ such that $\mathfrak{B} \uparrow \tau_{\mathfrak{M}} \cong \pi_{a}^{m} \mathfrak{M}$ and $\mathfrak{B}\left\lceil\tau_{\mathfrak{N}} \cong \pi_{\alpha}^{n} \mathfrak{N}\right.$. Therefore, $\mathfrak{B}\left\lceil\tau_{\mathfrak{M}} \sim_{\alpha} \mathfrak{M}\right.$ and $\mathfrak{B}\left\lceil\tau_{\mathfrak{N}} \sim_{\alpha} \mathfrak{N}\right.$, as required.

We prove that $\sim_{\alpha} \neq \sim_{\beta}$ for all ordinals $\alpha<\beta$. Noting that $\mathbf{Z} \sim_{\beta} \pi_{\beta} \mathbf{Z}$, it suffices to disprove $\mathbf{Z} \sim_{\alpha} \pi_{\beta} \mathbf{Z}$. Indeed, if this were the case, then for some $m, n \in \omega$ we would have $\left|\pi_{\alpha}^{m} \mathbf{Z}\right|=\left|\pi_{\alpha}^{n} \pi_{\beta} \mathbf{Z}\right|$. On the other hand we have

$$
\left|\pi_{\alpha}^{m} \mathbf{Z}\right| \leq \mu_{\alpha} \leq \sup _{\gamma<\beta} \mu_{\gamma}<\lambda_{\beta}=\left|\pi_{\beta} \mathbf{Z}\right| \leq\left|\pi_{\alpha}^{n} \pi_{\beta} \mathbf{Z}\right|
$$

a contradiction.

Finally, for each denumerable $\mathfrak{M}$, letting $M$ be the universe of $\mathfrak{M}$ we have $\left|\pi_{\alpha} \mathfrak{M}\right|=\left|\pi_{\alpha} M\right|=\left|\pi_{\alpha} \mathbf{Z}\right|>\omega$, and $\pi_{\alpha} \mathfrak{M} \sim_{\alpha} \mathfrak{M}$.

A family $F$ of limit ultrapowers is said to be commuting (with respect to composition) if and only if for every $\pi_{1}, \pi_{2} \in F$ we have $\pi_{1} \pi_{2} \mathfrak{M} \cong \pi_{2} \pi_{1} \mathfrak{M}$ for all structures $\mathfrak{M}$. The following is a simple variant of $[\mathbf{3}, 3.3]$.

COROLLARY. Let $S$ be a commuting semigroup of limit ultrapowers. Let $\sim_{S}$ be defined by $\mathfrak{M} \sim_{S} \mathfrak{N}$ if and only if $\pi_{1} \mathfrak{M} \cong \pi_{2} \mathfrak{N}$ for some $\pi_{1}, \pi_{2} \in S$. Then $\sim_{S}$ is a Robinson equivalence relation.

PrOOF. The proof follows from the final arguments in the above proof. Commutativity and the semigroup property are used to show that $\sim_{S}$ is transitive.

EXAMPLES. (i) The simplest commuting semigroups of limit ultrapowers are those of the form $S_{\pi}=\left\{\pi^{n} \mid n \in \omega\right\}$ for $\pi$ a fixed limit ultrapower. Using some kind of global choice principle if necessary, we see that every commuting family of limit ultrapowers can be extended to a maximal commuting semigroup.

(ii) As a further generalization, let us consider a semigroup $S$ of limit ultrapowers with the following property: for all $\pi_{1}, \pi_{2} \in S$ there are $\pi_{3}, \pi_{4} \in S$ such that

$$
\pi_{3} \pi_{1} \mathfrak{M} \cong \pi_{4} \pi_{2} \mathfrak{M}, \quad \text { for all structures } \mathfrak{M} \text {. }
$$

Defining $\sim_{S}$ as above, we still have that $\sim_{S}$ is a Robinson equivalence relation. Again, as in (i), $S$ can be extended to a maximal semigroup with property (*). 
(iii) Allowing $\pi_{3}$ and $\pi_{4}$ in $(*)$ to depend on the cardinality of $\mathfrak{M}$, one gets the most general possible Robinson equivalence relation, as proved by Lipparini in [3, 3.1 and 3.4].

\section{REFERENCES}

1. J. Barwise and S. Feferman (eds.), Model-theoretic logics, Perspectives in Math. Logic, Springer-Verlag, Berlin and New York, 1985.

2. C. C. Chang and H. J. Keisler, Model theory, 2nd ed., North-Holland, Amsterdam, 1977.

3. P. Lipparini, Robinson equivalence relations through limit ultrapowers, Boll. Un. Mat. Ital. B (6) 4 (1985), 569-583.

4. D. Mundici, An algebraic result about soft model theoretical equivalence relations, with an application to H. Friedman's fourth problem, J. Symbolic Logic 46 (1981), 523-530.

5. __ Duality between logics and equivalence relations, Trans. Amer. Math. Soc. 270 (1982), 111-129.

6. __ Embedding, amalgamation and elementary equivalence: the representation of compact logics, Fund. Math. 124 (1984), 109-122.

7. __ Abstract model theory and nets of $C^{*}$-algebras: noncommutative interpolation and preservation properties, Lecture Notes in Math., vol. 1103, Springer-Verlag, Berlin and New York, 1984, pp. 351-377.

8. (1986), 15-63.

9. M. E. Nadel, An arbitrary equivalence relation as elementary equivalence in an abstract logic, $\mathrm{Z}$. Math. Logik 26 (1980), 103-109.

Mathematics Institute "U. Dini" of The University, Loc. Romola N. 76, 50060 DONNINI, FLORENCE, ITALY 